\title{
Espermocultivo: crecimiento bacteriano del eyaculado y su relación con los parámetros seminales
}

\author{
Jenniffer Puerta Suárez ${ }^{1}$, Aracelly Villegas Castaño², Gabriel J. Serna Quintana ${ }^{3}$, \\ Alonso Martínez ${ }^{2}$, Johanna Romero Palacio ${ }^{3}$, Mariluz Giraldo 4 , Ángela Cadavid 1 , \\ Walter Cardona Maya. ${ }^{1}$ \\ ${ }^{1}$ Grupo Reproducción, ${ }^{2}$ Grupo Bacterias y Cáncer, Facultad de Medicina, Universidad de Antioquia. ${ }^{3}$ Laboratorio Clínico \\ PROLAB S.A.S. ${ }^{4}$ Escuela de Microbiología, Universidad de Antioquia. Colombia.
}

\section{RESUMEN}

Antecedentes: En el semen, algunos microorganismos pueden encontrar las condiciones óptimas para sobrevivir, ocasionando daños a los espermatozoides y desencadenando procesos de infertilidad o infecciones del tracto reproductivo. Entender el papel de los microorganismos aislados en el semen, contribuye a mejorar el diagnóstico de casos de infertilidad donde la única causa aparente son los procesos infecciosos. Objetivo: Describir y correlacionar los parámetros seminales y el crecimiento bacteriano del eyaculado. Métodos: Identificación de los microorganismos aislados en 43 espermocultivos-clínicos y 28 espermocultivos-investigación. Se realizó conteo de las unidades formadoras de colonia a los espermocultivos-investigación y análisis de las características espermáticas. Resultados: Se obtuvo crecimiento bacteriano en $14(32,6 \%)$ de los espermocultivos-clínicos y 15 (53,6\%) de los espermocultivos-investigación. Los microorganismos aislados fueron Enterococcus faecalis, Escherichia coli, Morganella morganii, Staphylococcus spp coagulasa negativo, Klebsiella pneumoniae y microbiota mixta. En este estudio se observó abundante crecimiento de cocos aerobios. Finalmente, no se encontró asociación entre la disminución en la calidad de los parámetros seminales y los microorganismos. Conclusiones: La presencia de bacterias en el semen no afecta la calidad seminal.

\section{PALABRAS CLAVE: Espermatozoides, bacterias, infección, microbiota, cultivo de semen}

\section{SUMMARY}

Background: Microorganisms can find the optimal conditions for survival in semen, causing damage to the spermatozoa and triggering processes of infertility or reproductive tract infections. Therefore, understanding the role of the microorganisms present in semen can help to improve the diagnosis of infertility cases where the only apparent cause is infectious processes. Objective: To describe and correlate semen parameters and bacterial growth in ejaculate. Methods: Identification of microorganisms isolated in 43 clinical spermocultures and 28 research spermocultures. We assessed colony-forming unit counts and sperm characteristics of research spermocultures. In addition, semen parameters were evaluated in each ejaculate. Results: Bacterial growth was obtained in $14(32.6 \%)$ of the 43 clinical spermocultures and $15(53.6 \%)$ of the 28 research spermocultures. The isolated microorganisms were Enterococcus faecalis, Escherichia coli, Morganella morganii, Staphylococcus coagulase negative, Klebsiella pneumoniae and mixed microbiota. Finally, in this study a large growth of aerobic cocci was observed. We did not find association between the decline in the quality of semen parameters and microorganisms. Conclusion: The presence of bacteria in semen does not affect semen quality.

KEY WORDS: Spermatozoa, bacteria, infection, microbiota, spermocultures 


\section{INTRODUCCIÓN}

Los microorganismos habitan gran diversidad de ambientes y colonizan a los seres humanos. Producto de esta interacción, los humanos se adaptan a ciertos estímulos; sin embargo, no todas estas relaciones son benéficas (1). El contenido y la variedad de la microbiota que nos defiende de infecciones por microorganismos patógenos y nos ayuda al metabolismo de nutrientes (2), difiere entre hombres y mujeres, característica que está relacionada con las diferencias anatómicas, por ejemplo, en el caso de la uretra, la cual mide hasta cinco veces más en los hombres y contiene abundantes cantidades de IgA, IgG e IgM, forman una barrera de defensa y evitan la adhesión e invasión microbiana. Estas características permiten explicar porque en las mujeres se presenta mayor frecuencia de infecciones urinarias (3).

El tracto urinario tiene mecanismos de defensa contra la colonización por determinados microorganismos: la descamación, la orina y los péptidos antimicrobianos (urea, IgA y factores antiadhesinas como la proteína de Tamm-Horsfall) (4). De otro lado, la alta concentración de zinc y de péptidos antimicrobianos como fosfolipasa A, lisozima, espermina y espermidina presentes en las secreciones prostáticas o en el plasma seminal, inhiben el crecimiento bacteriano $(3,5)$.

La microbiota uretral y del tracto reproductivo está determinada por factores como la madurez y la actividad sexual, el número de compañeros(as) y el tipo de relación sexual (anal, oral o genital) (3). En los individuos no circuncidados, el prepucio favorece la humedad y reduce la descamación del espacio prepucial convirtiéndose en un área cálida y húmeda con un $\mathrm{pH}$ neutro o ligeramente alcalino y con concentraciones de oxígeno reducidas (3), condiciones idóneas para el crecimiento bacteriano y viral (6).

En contraste, la infertilidad, que afecta al $10 \%$ de las parejas de la población general, en donde el factor masculino juega un papel importante, se ha observado que del 8 al $35 \%$ de los problemas se relacionan con infecciones que causan obstrucción, daño celular, daño testicular, epididimitis y orquitis $(7,8)$. Un ejemplo, de lo anterior son las infecciones por Chlamydia trachomatis que generan inflamación de la glándula prostática y epididimitis. Sin embargo, los efectos de esta infección sobre la calidad espermática no son claros (7, 9-11), mientras que algunos estudios demuestran que esta infección reduce la movilidad, la viabilidad, la concentración y la morfología espermáticas $(9,11)$, otros investigadores no reportan ningún efecto deletéreo sobre la calidad seminal (10). Finalmente, se plantea si el espermatozoide humano sirve como vehículo para transportar agentes patógenos al tracto reproductivo femenino, como ocurre con el virus de la inmunodeficiencia humana (VIH) (12-15).

En el semen, algunos microorganismos encontrarían las condiciones óptimas para sobrevivir, esto ocasionaría daños a los espermatozoides después de la eyaculación por algún efecto directo sobre la membrana plasmática espermática o por efectos indirectos inducidos por los factores solubles producto del metabolismo bacteriano, desencadenando procesos de infertilidad o infecciones en el tracto reproductivo femenino. Por lo tanto, determinar los efectos que tienen los microorganismos al estar en contacto con el semen, contribuiría a mejorar el diagnóstico de casos de infertilidad donde la única causa aparente son los procesos infecciosos.

El objetivo del presente trabajo es describir la presencia de bacterias en el semen humano, además de correlacionar los parámetros seminales y el crecimiento bacteriano del eyaculado.

\section{PACIENTES Y MÉTODOS}

Se realizó una descripción de los microorganismos aislados en espermocultivos-clínicos de 43 muestras obtenidas de pacientes entre los años 2007 y 2013 remitidos por el médico tratante al Laboratorio Clínico Prolab S.A.S. Medellín, Colombia (http://www.prolab.com.co). Las muestras se cultivaron en agar sangre, chocolate y MacConkey, e incubadas durante 72 horas a $37^{\circ} \mathrm{C} / 5 \% \mathrm{CO} 2$. En las muestras con crecimiento bacteriano, se determinó el género y la especie del germen aislado y el perfil de resistencia a antibióticos. A la muestra inicial se le realizó un extendido para ser coloreado empleando la tinción de Gram.

Posteriormente, se realizaron 28 espermocultivos-investigación en las instalaciones del Grupo Reproducción, Facultad de Medicina, Universidad de Antioquia, Medellín, Colombia, entre mayo de 2013 y agosto de 2014. Las muestras obtenidas por masturbación fueron donadas por voluntarios aparentemente sanos en un recipiente estéril luego de dos a cinco días de abstinencia sexual. El promedio de edad de los voluntarios fue de 29 años (rango: 20 a 56 años) y cada individuo firmó un consentimiento informado aprobado por el Comité de Ética de la Universidad de Antioquia, después de leer y entender el propósito de la investigación. Se incluyeron 19 voluntarios con fertilidad desconocida, 5 voluntarios fértiles, 2 voluntarios con diagnóstico de varicocele y 2 infértiles, en este último grupo, un voluntario se había practicado la vasectomía. Después del proceso de licuefacción, 10 $\mu \mathrm{L}$ del eyaculado se sembraron en agar chocolate por el método de agotamiento, los cultivos se incubaron durante 24 a 48 horas a $37^{\circ} \mathrm{C} / 5 \%$ de $\mathrm{CO} 2$. 
Posteriormente se realizó el conteo de las unidades formadoras de colonias (UFC) de cada muestra y los microorganismos aislados se identificaron por pruebas bioquímicas. Paralelamente, a cada muestra de semen se le realizó la evaluación de los parámetros seminales de acuerdo a los lineamientos de la Organización Mundial de la Salud (OMS) en su Manual de Laboratorio para la Examinación y Procesamiento del Semen Humano del 2010 (16), excepto la concentración espermática que fue determinada usando la cámara de Makler (17).

El análisis estadístico se realizó mediante estadística descriptiva empleando la mediana y el rango intercuartílico representando cada variable. Se aplicó el test de Kruskal Wallis para las comparaciones entre los diferentes grupos en el programa estadístico Prism 5.0 (GraphPad Software, San Diego,CA).

\section{RESULTADOS}

De los 43 espermocultivos-clínicos de muestras de semen realizados entre los años 2007 y 2013, catorce $(32,6 \%)$ presentaron crecimiento bacteriano. Las especies más frecuentes fueron $\mathrm{E}$. faecalis $(n=4)$, M. morganii $(n=1)$, E. coli $(n=3)$ y crecimiento mixto de bacterias $(n=6)$. La edad promedio de los pacientes de este grupo fue de 38 años (rango: 20 a 66 años).

A través de la coloración de Gram se detectó la presencia de microorganismos en 13 muestras, de las cuales se confirmó la presencia del agente infeccioso por cultivo en 5 muestras; en las restantes el cultivo no permitió aislar ningún microorganismo. Se observó reacción leucocitaria en 7 del total de las muestras, en 3 de ellas hubo crecimiento bacteriano y se observó presencia de microorganismos al Gram, en las 4 restantes no fue posible correlacionar la reacción leucocitaria con la presencia de algún microorganismo, ni en el cultivo ni al Gram. En los cultivos con crecimiento mixto no se realizó identificación de los microorganismos ni antibiograma.

Los antibiogramas para los aislamientos de $\mathrm{E}$. faecalis fueron sensibles a ampicilina, nitrofurantoina y ciprofloxacina. Los cultivos con crecimiento de E. coli presentaron sensibilidad a las cefalosporinas, los carbapenems, aztreonam, ciprofloxacina y gentamicina. El aislamiento de M. morganii fue sensible a los antibióticos amikacina, cefepime, ciprofloxacina, gentamicina y a los carbapenems.

De los espermocultivos-investigación realizados en 28 voluntarios en el Grupo Reproducción, el $53,6 \%(n=15)$ presentó crecimiento de algún microorganismo. El promedio de recuento bacteriano fue de 7,1 x 103 UFC/mL de eyaculado (rango entre 0 a 5x104UFC/mL). En la Tabla I se describen los
Tabla I

MICROORGANISMOS AISLADOS EN LOS ESPERMOCULTIVOSY SU FRECUENCIA

\begin{tabular}{lcc}
\hline Microorganismo & \multicolumn{2}{c}{ Espermocultivo } \\
& Clínico & Investigación \\
\hline Enterococcus faecalis & 4 & 1 \\
Escherichia coli & 3 & 1 \\
Morganella morganii & 1 & - \\
Klebsiella pneumoniae & - & 1 \\
Staphylococcus spp. & - & 8 \\
coagulasa negativo & & 4 \\
Microbiota mixta & 6 & 15 \\
\hline Total & 14 & \\
\hline
\end{tabular}

aislamientos bacterianos provenientes de los espermocultivos clínicos y de investigación.

Las Figuras 1-4 muestran la mediana y el rango intercuartílico para los parámetros seminales: volumen (Figura 1), concentración (Figura 2), viabilidad (Figura 3) y movilidad tanto tipo I como tipo II (Figura 4) de los cuatro grupos: hombres con fertilidad desconocida, hombres fértiles, hombres infértiles y hombres con varicocele de las muestras positivas (+) y negativas (-) para el cultivo.

En la Figura 1, se observa que en el grupo de los hombres fértiles con cultivos positivos hay un aumento en el volumen seminal respecto a los fértiles con cultivo negativo, esta situación es inversa en los hombres con varicocele. Tanto en los hombres fértiles como en los infértiles, la concentración espermática es mayor en quienes presentan crecimiento bacteriano en el eyaculado, contrario a lo que sucede en los hombres con varicocele y en hombres de la población con fertilidad desconocida (Figura 2). En los individuos de los grupos de hombres fértiles, infértiles y con varicocele que presentan crecimiento bacteriano en el eyaculado también presentan mayor movilidad espermática (Figura 3). La viabilidad espermática fue mayor en los hombres fértiles e infértiles con cultivos positivos (Figura 4) y el número de UFC/mL de eyaculado fue mayor en los infértiles que en los otros grupos, seguido de los hombres fértiles y con varicocele y por último los voluntarios de la población con fertilidad desconocida (Figura 5). Sin embargo, no se encontró diferencia estadísticamente significativa entre los parámetros seminales de los individuos con cultivos positivos comparados con los negativos. 
volumen

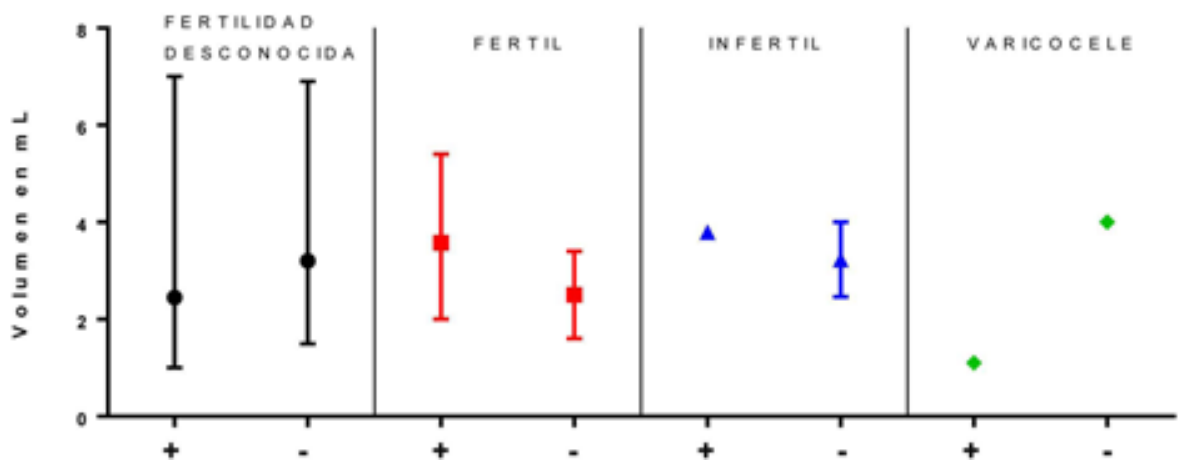

Figura 1. Volumen seminal y su relación con el crecimiento bacteriano del eyaculado.

\section{concentración espermática}

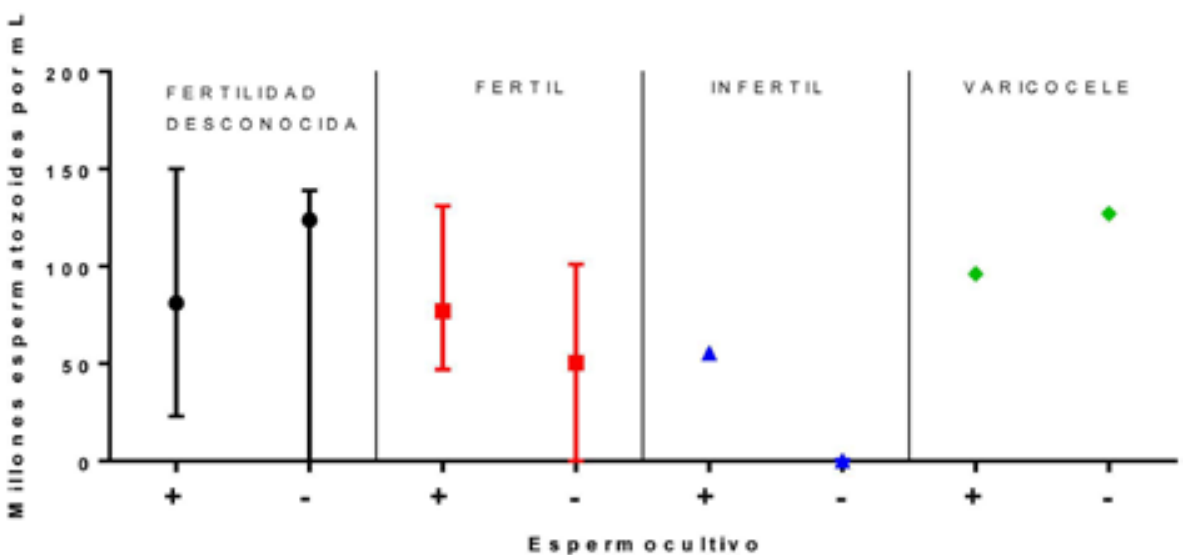

Figura 2. Concentración espermática y su relación con el crecimiento bacteriano del eyaculado.

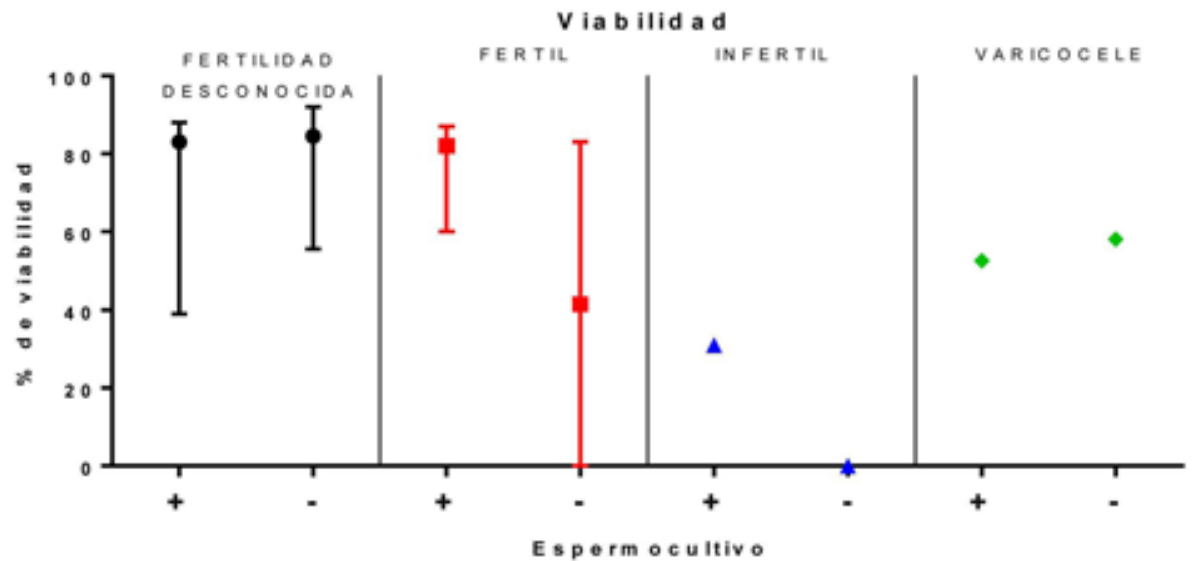

Figura 3. Viabilidad espermática, relación con el crecimiento bacteriano del eyaculado. Viabilidad espermática, relación con el crecimiento bacteriano del eyaculado. 


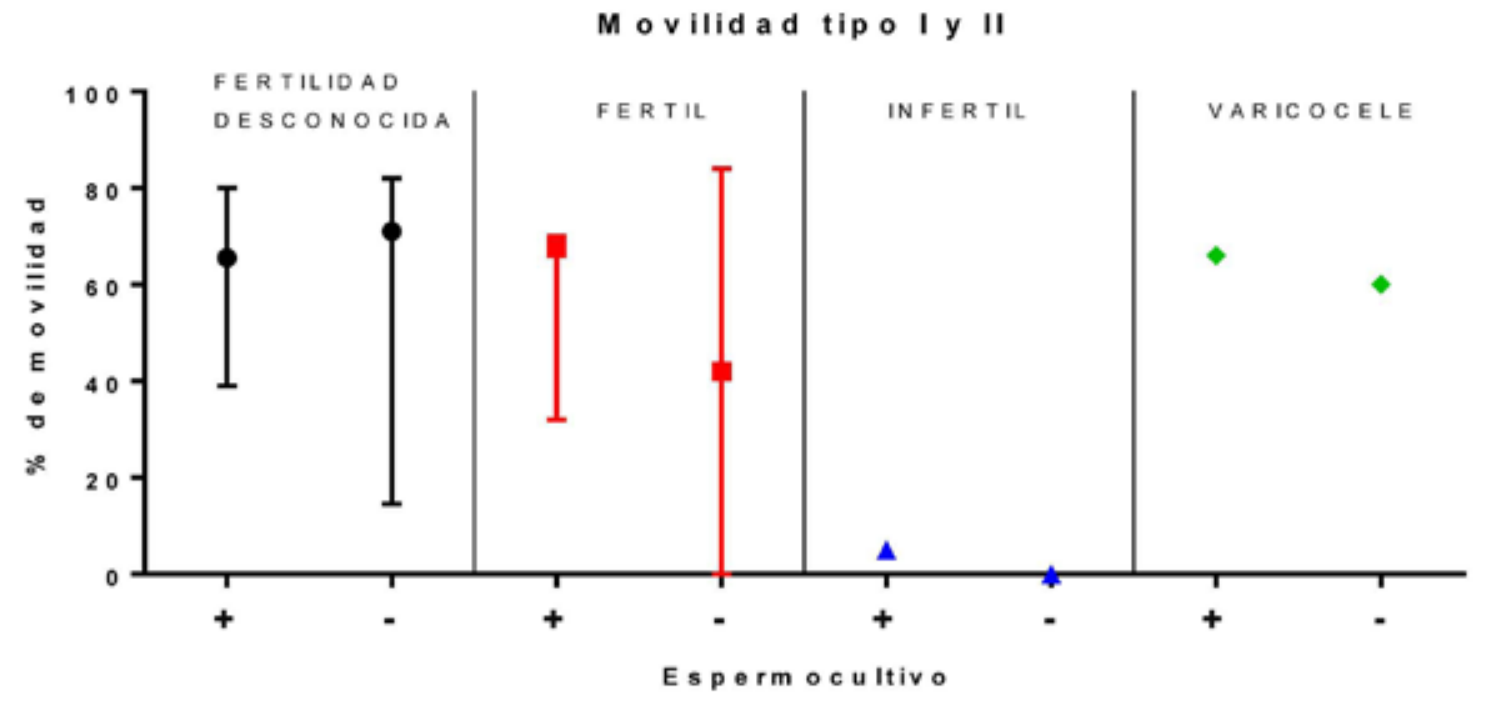

Figura 4. Movilidad I más II, relación con el crecimiento bacteriano del eyaculado.

Crecim ien to b a cteriano

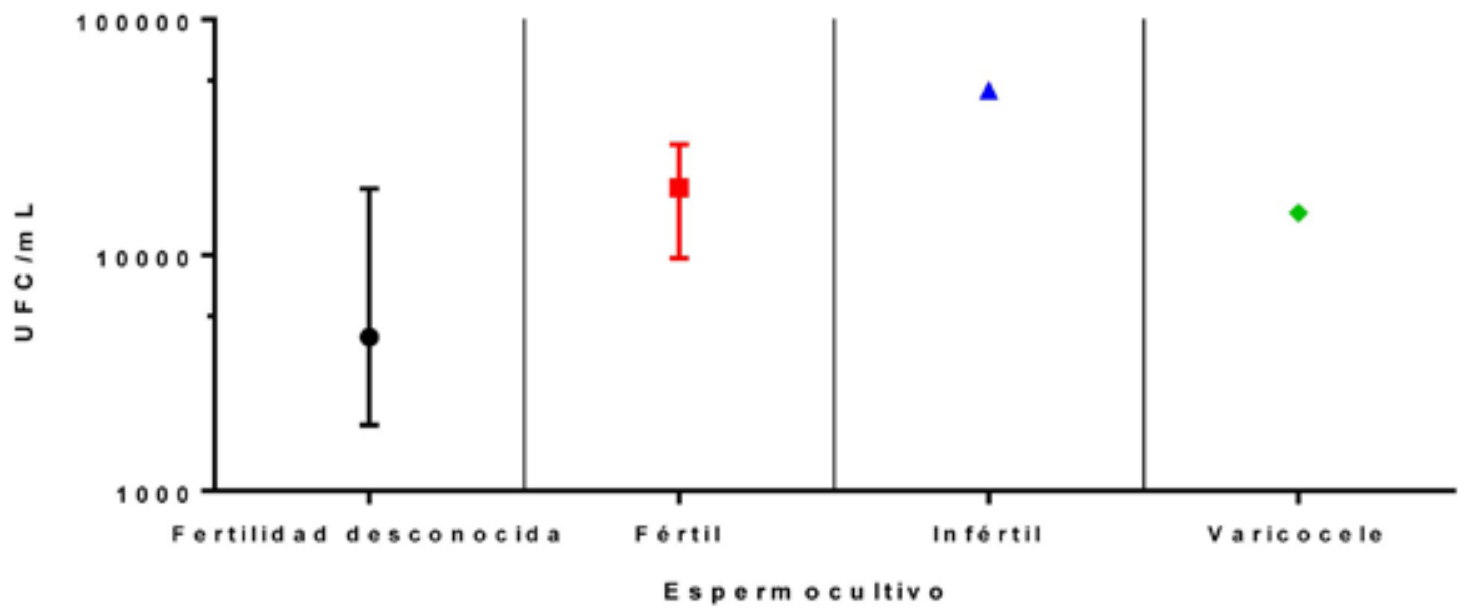

Figura 5. Unidades formadoras de colonia por $\mathrm{mL}$ de eyaculado.

Con relación a los parámetros seminales, un donante infértil presentó oligoastenoteratozoospermia y el cultivo bacteriano fue negativo; un individuo con fertilidad desconocida presentó disminución en el volumen; 4 individuos presentaron azoospermia: un fértil, un infértil, un individuo con fertilidad desconocida y como era de esperarse el voluntario vasectomizado. Un individuo del grupo con fertilidad desconocida y uno del grupo de infértiles presentaron disminución de la movilidad espermática. Los 20 individuos restantes presentaron parámetros seminales normales según el límite inferior de referencia propuesto por la OMS (16). 


\section{DISCUSIÓN}

El presente estudio describe la presencia de microorganismos presentes en el semen, inclusive en hombres con fertilidad probada, sin afectar las características seminales y su presencia puede estar asociada a contaminaciones provenientes de la microbiota uretral o de la piel, los cuales no necesariamente serían causantes de procesos infecciosos. Estos resultados coinciden parcialmente con los datos obtenidos por Moretti y cols, quienes reportan que la contaminación bacteriana seminal es catalogada como frecuente, sin embargo, estos autores encontraron que los microorganismos contribuyen al deterioro de la calidad espermática (18).

Durante la eyaculación, los espermatozoides pasan a través de los conductos eyaculadores y se mezclan con los fluidos de la vesícula seminal, la próstata y las glándulas bulbouretrales para formar el semen que es transportado hasta la uretra, el cual ha sido catalogado como un medio para la transmisión de microorganismos entre hombres y mujeres, e incluso algunos de éstos pueden interferir con el éxito reproductivo (2). Entre los principales microorganismos que afectan la calidad espermática se incluyen $\mathrm{E}$. coli $(18,19)$, E. faecalis (20), U. urealyticum $(21,22)$, N. gonorrhoeae, C. trachomatis $(9,11)$ y $\mathrm{M}$. hominis $(23)$.

Este estudio encontró crecimiento bacteriano que demuestra que el semen no es un fluido completamente estéril y muchas de estas bacterias están relacionadas con la microbiota de la uretra, la orina y la piel, lo que sugiere que éstas en el semen tendrían múltiples orígenes, entendiendo como microbiota los microorganismos que usualmente habitan una zona anatómica, donde se multiplican y conviven con su huésped sin causarle daño o incluso beneficiándolo. El tracto reproductivo masculino es un sitio que presenta una gran diversidad con relación a la microbiota, por ejemplo, se conoce que de allí se logra aislar Staphylococcus coagulasa negativos, S. aureus, Streptococcus del grupo viridans, Corynebacterium spp, Streptococcus $\beta$-hemolíticos y Enterobacter spp (3).

Existen varios métodos para estudiar la presencia de bacterias en el semen, no obstante, presentan limitantes como la dificultad en el cultivo de algunos microorganismos, la caracterización de la microbiota y el proceso de obtención de la muestra, por ejemplo, el diagnóstico de prostatitis se realiza empleando la prueba de los cuatro vasos de Meares y Stame y que incluye un masaje prostático con obtención de muestras fraccionadas de orina antes y después del procedimiento y líquido seminal, pero algunos pacientes rechazan este tipo de procedimientos (24).

El espermocultivo surge como una alternativa al diagnóstico de procesos infecciosos del tracto reproductivo masculino a pesar de presentar un alto subdiagnóstico de entidades como la prostatitis bacteriana. Un estudio de Mendoza y cols (24), evaluó la sensibilidad del espermocultivo en el diagnóstico de prostatitis bacteriana crónica y encontró que la técnica tiene una sensibilidad de sólo el 10\% comparada con la prueba de Meares y Stamey, donde el microorganismo más aislado fue S. aureus, seguido por E. coli, E. aglomerans, E. faecalis, K. oxytoca, Citrobacter freundi, P. vulgaris y S. epidermidis.

Diferentes grupos bacterianos en alto número, están presentes en las muestras de semen tanto de individuos fértiles, infértiles y de voluntarios sanos, sin encontrar diferencias significativas entre los grupos analizados, sin embargo, otros estudios como el realizado por Hou y cols (25), reportan que la presencia de bacterias como Anaerococcus spp. afecta la calidad seminal y se ha descrito que las infecciones agudas y crónicas pueden generar inflamación en el tracto reproductivo masculino afectando la espermatogénesis (26); además se ha propuesto que cada vez más hombres infértiles sufren de inflamación aguda o crónica del tracto genitourinario, en ausencia de síntomas (22). Bacterias anaerobias estrictas como Anaerococcus spp son cultivadas en medios diferentes a los usados en este estudio y en atmósfera de anaerobiosis, esto podría explicar porqué se observan microorganismos en el Gram pero no se logran cultivar en las condiciones aquí empleadas. Otra explicación posible a la presencia de bacterias en el Gram no confirmadas por cultivo es el inicio de terapia antibiótica previa toma de la muestra.

Aunque muchos autores reportan disminución de la calidad en los parámetros seminales en individuos con infecciones clínicas o subclínicas $(9,11,27)$ esta interacción es aun controversial. En pacientes con prostatitis crónica son aisladas bacterias como E. coli, C. freundi, S. aureus, E. faecalis y S. epidermidis (28); algunos de éstos encontrados en las muestras analizadas en este estudio, sin embargo, la cantidad de UFC difiere, porque se considera infección cuando se obtienen más de 105 UFC. No obstante, solo se realizó búsqueda de microorganismos aerobios, por lo que queda por definir el papel de los agentes anaerobios e intracelulares.

En este estudio se observó crecimiento de cocos aerobios que acorde con lo reportado por 
Metha y cols corresponden al $50 \%$ de las bacterias presentes en muestras de semen, en especial de hombres de parejas infértiles (29).

\section{CONCLUSIÓN}

Aunque se encontró asociación entre la disminución en la calidad de los parámetros seminales y los microorganismos, no se puede descartar que esta relación dependa del microorganismo presente en el eyaculado o quizás del número de muestras analizadas, por lo que la presencia de bacterias anaerobias e intracelulares debe ser investigada.

Agradecimientos: Por el apoyo financiero otorgado por COLCIENCIAS (111556933373) y a la Estrategia de Sostenibilidad 2014-2015 Grupo Reproducción, JPS fue Joven Investigadora de Colciencias.

\section{REFERENCIAS}

1. Mandar R. Microbiota of male genital tract: impact on the health of man and his partner. Pharmacol Res 2013;69(1):32-41.

2. Puerta-Suárez J, Giraldo M, Cadavid ÁP, CardonaMaya W. Infecciones bacterianas del tracto reproductivo masculino y su papel en la fertilidad. Rev Chil Obstet Ginecol 2014;79(3):209-17.

3. Wilson M. Bacteriology of humans: an ecological perspective: John Wiley \& Sons; 2009.

4. Chromek M, Slamová Z, Bergman P, Kovács L, Podracká Lu, Ehrén I, et al. The antimicrobial peptide cathelicidin protects the urinary tract against invasive bacterial infection. Nat Med 2006;12(6):636-41.

5. Malm J, Sørensen O, Persson T, Frohm-Nilsson M, Johansson B, Bjartell A, et al. The human cationic antimicrobial protein (hCAP-18) is expressed in the epithelium of human epididymis, is present in seminal plasma at high concentrations, and is attached to spermatozoa. Infec Immun 2000;68(7):4297-302.

6. Cardona-Arias J, Puerta-Suárez J, Flórez-Duque J. Prevalencia del virus papiloma humano y sus factores de riesgo en hombres: revisión sistemática. Infectio 2011;15(4):268-76.

7. Liu J, Wang Q, Ji X, Guo S, Dai Y, Zhang Z, et al. Prevalence of Ureaplasma urealyticum, Mycoplasma hominis, Chlamydia trachomatis infections, and semen quality in infertile and fertile men in China. Urology 2014;83(4):795-9.

8. Pellati D, Mylonakis I, Bertoloni G, Fiore C, Andrisani $A$, Ambrosini $G$, et al. Genital tract infections and infertility. Eur J Obstet Gynecol Reprod Biol 2008;140(1):311.

9. Molano M, Weiderpass E, Posso H, Morre SA, Ronderos $\mathrm{M}$, Franceschi $\mathrm{S}$, et al. Prevalence and determinants of Chlamydia trachomatis infections in women from Bogota, Colombia. Sex Transm Infect 2003;79(6):474-8.
10. Zaneveld LJ, Waller DP, Anderson RA, Chany C, 2nd, Rencher WF, Feathergill K, et al. Efficacy and safety of a new vaginal contraceptive antimicrobial formulation containing high molecular weight poly(sodium 4-styrenesulfonate). Biol Reprod 2002;66(4):886-94.

11. Dohle GR. Inflammatory-associated obstructions of the male reproductive tract. Andrologia 2003;35(5):321-4.

12. Cardona Maya W, Rugeles M, Cadavid AP. [Interactions between human spermatozoids and human immunodeficiency virus]. Actas Urol Esp 2009;33(3):223 $-6$.

13. Cardona-Maya W, Velilla PA, Montoya CJ, Cadavid A, Rugeles MT. In vitro human immunodeficiency virus and sperm cell interaction mediated by the mannose receptor. J Reprod Immunol 2011;92(1-2):1-7.

14. Cardona-Maya W, Velilla P, Montoya CJ, Cadavid A, Rugeles MT. Presence of HIV-1 DNA in spermatozoa from HIV-positive patients: changes in the semen parameters. Current HIV Res 2009;7(4):418-24.

15. Cardona-Maya W, Lopez-Herrera A, Velilla-Hernandez P, Rugeles MT, Cadavid AP. The role of mannose receptor on HIV-1 entry into human spermatozoa. Am J Reprod Immunol 2006;55(4):241-5.

16. WHO. WHO laboratory manual for the examination and processing of human semen. Fifth edition, 2010. Disponible en: http://whqlibdoc.who.int/publications/2010/9789241547789_eng.pdf.

17. Cardona-Maya W, Berdugo J, Cadavid A. [Comparing the sperm concentration determined by the Makler and the Neubauer chambers]. Actas Urol Esp 2008;32(4):443-5.

18. Moretti E, Capitani S, Figura N, Pammolli A, Federico MG, Giannerini $\mathrm{V}$, et al. The presence of bacteria species in semen and sperm quality. J Assis Reprod Genet 2009;26(1):47-56

19. Diemer T, Weidner W, Michelmann H, Schiefer HG, Rovan E, Mayer F. Influence of Escherichia coli on motility parameters of human spermatozoa in vitro. Int J Androl 1996;19(5):271-7.

20. Merino G, Carranza-Lira S, Murrieta S, Rodriguez L, Cuevas E, Moran C. Bacterial infection and semen characteristics in infertile men. Arch Androl 1995;35(1):43-7.

21. Toth $A$, Lesser ML. Ureaplasma urealyticum and infertility: the effect of different antibiotic regimens on the semen quality. J Urol 1982;128(4):705-7.

22. Fraczek M, Kurpisz M. Inflammatory mediators exert toxic effects of oxidative stress on human spermatozoa. J Androl 2007;28(2):325-33.

23. Gdoura R, Kchaou W, Chaari C, Znazen A, Keskes L, Rebai T, et al. Ureaplasma urealyticum, Ureaplasma parvum, Mycoplasma hominis and Mycoplasma genitalium infections and semen quality of infertile men. BMC Infec Dis 2007;7(1):129.

24. Mendoza Díaz N, Aguirre Castañeda R, Del Castillo Mori A. Evaluación de la sensibilidad del cultivo de semen en el diagnóstico de prostatitis bacteriana crónica. Rev Med Hered 2004;15(1):37-43.

25. Hou D, Zhou X, Zhong X, Settles ML, Herring J, Wang $\mathrm{L}$, et al. Microbiota of the seminal fluid from healthy and infertile men. Fertil Steril 2013;100(5):1261-9.

26. Sanocka-Maciejewska D, Ciupińska M, Kurpisz M. 
Bacterial infection and semen quality. J Reprod Immunol 2005;67(1):51-6.

27. Sanocka-Maciejewska D, Ciupinska M, Kurpisz $M$. Bacterial infection and semen quality. J Reprod Immunol 2005;67(1-2):51-6.

28. Mendoza Díaz N. Evaluación de la sensibilidad del cultivo de semen en el diagnóstico de prostatitis bacteriana crónica. Rev Med Hered 2004;15(1):3743.

29. Mehta R, Sridhar H, Kumar B, Kumar T. High incidence of oligozoospermia and teratozoospermia in human semen infected with the aerobic bacterium Streptococcus faecalis. Reprod Biomed online 2002;5(1):1721 